\title{
ANALYSIS OF THE INHIBITORY POTENTIAL OF CLINICAL STREPTOCOCCUS SALIVARIUS ISOLATES FROM SALIVA AND DORSAL OF TONGUE OF ADULTS ON ENTEROCOCCAL FAECALIS GROWTH
}

\author{
YOHANES BOSKO ARDY WINOTO, HEDIJANTI JOENOES, BOY M BACHTIAR*
}

Department of Oral Biology, Faculty of Dentistry, Universitas Indonesia, Jakarta, Indonesia. Email: boybachtiar@gmail.com Received: 21 April 2017, Revised and Accepted: 13 July 2017

\section{ABSTRACT}

Objective: The aim of this study is to analyze the inhibitory potential of Streptococcus salivarius isolates from the saliva and dorsum of the tongue of adults on Enterococcal faecalis American Type Culture Collection (ATCC) 29212.

Methods: Deferred antagonism and agar well diffusion methods were used to analyze the potential of $S$. salivarius to inhibit $E$. faecalis growth with S. salivarius ATCC 13419 as the positive control.

Result: The maximum inhibitor diameter for each isolation was $11.17 \mathrm{~mm}$ at $10_{10} \mathrm{CFU}$ for the dorsum of tongue isolates and $8.17 \mathrm{~mm}$ at $10^{9} \mathrm{CFU}$ for saliva isolates.

Conclusions: Clinical S. salivarius isolates from the dorsum of tongue had greater potential for inhibiting E. faecalis growth compared to the saliva isolates and control bacteria.

Keywords: Probiotic, Streptococcus salivarius, Enterococcal faecalis, Deferred antagonism method, Agar well diffusion method.

(C) 2017 The Authors. Published by Innovare Academic Sciences Pvt Ltd. This is an open access article under the CC BY license (http://creativecommons. org/licenses/by/4. 0/) DOI: http://dx.doi.org/10.22159/ajpcr.2017.v10s5.23080

\section{INTRODUCTION}

The World Health Organization defines probiotic agents as live microorganisms which, when applied in an adequate number, support the health of the human body [1]. Some types of bacteria have been widely used as probiotic agents, such as Enterococcus, Streptococcus, Escherichia, Lactobacillus, and Bifidobacterium as the bacteria most often used. Based on the previous studies of probiotics, Lactobacillus bacteria can be used as both a treatment and prevention of infections causing acute diarrhea of children. Lactococcus lactis could be used to deliver vaccine proteins and the DNA vaccines, and Streptococcus salivarius K12 can treat throat infections [2-4]. The bacteria listed above are probiotic agents that eliminate pathogens and produce similar proteins types. Lantibiotic proteins are small proteins that are modified during the post-translational phase and synthesized on ribosomes with clusters of lanthionin (Lan) and/or $\beta$-methyllanthionin (Melan) [5]. S. salivarius can produce lantibiotics [6].

S. salivarius is an indigenous bacterium that exists in the oral cavity of newborns. These bacteria facilitate biofilm formation and later move to the dorsal tongue, oral mucosa, and pharyngeal mucosa. S. salivarius also produces the secondary lantibiotic protein. Lantibiotics produced by some strains of $S$. salivarius can eliminate some harmful bacteria. Thus, the role of $S$. salivarius is very important to maintain the balance of microorganisms (agent) in the oral cavity. Dental disease is caused by an imbalance of host, agent, and environmental factors [5]. In the data obtained from the Indonesian National Health Survey in 2007, the prevalence of caries in Indonesia reached $72.1 \%$ with average active caries reaching $43.4 \%$ [7]. Dental caries is caused by specific types of bacteria that produce acid which demineralize and weaken the tooth structure [8]. Periodontitis was the second most common tooth and mouth disease in Indonesia. Periodontitis is a disease of the oral cavity which causes the tissues supporting the teeth to lose collagen, affecting $25.9 \%$ of the entire Indonesian population [7]. Most pathogens associated with periodontal disease are Gram-negative anaerobic rods, whereas some pathogens are also Gram-positive facultative and anaerobic cocci and rods, and Gram-negative facultative rods [9]. Both diseases are exacerbated by the presence of Enterococcal faecalis American Type Culture Collection (ATCC) 29212 in the oral cavity [10].

In dentistry, there are several alternative treatments for preventing the development of nosocomial infections due to E. faecalis. Sodium hypochlorite is an irrigation material that is often used, as well as chlorhexidine mouthwash. However, the research by Maria et al. suggested that complications from the use of sodium hypochlorite solution caused mucosal tissue irritation [11]. Several studies have established the inhibiting ability of $S$. salivarius as a probiotic agent to fight against pathogenic bacteria. However, observing $S$. salivarius derived from the saliva and dorsal tongues of healthy individuals against the growth of $E$. faecalis have not been tested, and there for being studied in this research.

\section{METHODS}

Preparation of subject samples

The participants in this study were adults with healthy teeth and mouths. Before sampling, the participants were asked to avoid foods that contained sugar, sour flavors, or high amounts of caffeine shortly before sampling, as it would interfere with the test by lowering the $\mathrm{pH}$ of saliva and increase the growth of bacteria. No food was consumed $1 \mathrm{hr}$ before sampling. A water rinse removed food debris, and the test participants waited up to 10 minutes after the rinse to avoid saliva dilution before sampling.

Sampling method of stimulated saliva and dorsal tongue smear Sampling included collecting $10 \mathrm{ml}$ of whole saliva by stimulating saliva production using Parafilm M. Participants were instructed to chew the Parafilm M for 10 minutes and collect saliva into a sterile tube. Saliva samples were sealed, and stored temporarily at $4^{\circ} \mathrm{C}$. Dorsal tongue swab sampling was done in the morning. Before sampling, tongue smears were collected using sterile cotton rolls. Dorsal tongue smear samples were collected using citobrushes from the circumvallated part 
of the tongue to the tip of the tongue. Then, the citobrush was placed in a vial that already contained phosphate buffered saline and was also stored temporarily at $4^{\circ} \mathrm{C}$.

\section{Identification method of $S$. salivarius}

The samples of saliva and dorsal tongue smears were taken and cultured on medium mitis Salivarius agar (MSA). The medium was then incubated anaerobically at a temperature of $37^{\circ} \mathrm{C}$ for $24 \mathrm{hrs}$. Afterward, colonies of the culture samples considered morphological $S$. salivarius (gum drop-like) were taken, and polymerase chain reaction (PCR) tests were conducted using S. salivarius ATCC 13419 as a positive control (Fig. 1). The PCR test began with the initial denaturation temperature of $94^{\circ} \mathrm{C}$ for 10 minutes and continued for 30 cycles. This was followed by an annealing temperature of $55^{\circ} \mathrm{C}$ for 30 seconds, and elongation temperature of $65^{\circ} \mathrm{C}$ using SalA US primers (5'-GTAGAAAATATTTACTACATACT) and salads (5'-GTTAAAGTATTCGTAAAACTGATG) [10]. PCR results were analyzed using $1 \%$ agarose electrophoresis for 30 minutes $(100 \mathrm{~V})$ [10] which showed positive results colony of $S$. salivarius, ATCC and clinically, then cultured in the medium brain-heart infusion (BHI) agar and BHI broth for $18 \mathrm{hrs}$

\section{Identification and calculation method protein concentration} S. salivarius

Clinical S. salivarius and ATCC colonies were taken and cultured in BHI broths for $18 \mathrm{hrs}$. Then, the pellet and supernatant of the bacterial cell lysates were separated using a buffer and centrifuge. The pellets were analyzed using sodium dodecyl sulfate polyacrylamide gel electrophoresis (SDS-PAGE) (150 V, 80 mA, 60 minutes) and Coomassie blue staining to see whether there was the same molecular mass (kDa) of the same protein produced by $S$. salivarius. SDS-PAGE testing was obtained for participants with the same protein profile. Later, colonies of $S$. salivarius with the same protein profiles were put in Eppendorf tubes containing $30 \%$ glycerol and stored at $-80^{\circ} \mathrm{C}$ to be used as stock

\section{Methods of analyzing potential inhibiting power of $S$. salivarius} $S$. salivarius' inhibitory potency against $E$. faecalis was tested using a deferred antagonism method. This method was performed on a medium BHI agar. S. salivarius and S. salivarius ATCC 13419 were diluted 10-1, $10-2$, and 10-3, respectively, into four different concentrations. Each concentration was inoculated in lines $1 \mathrm{~cm}$ apart on the BHI agar. The agar plates were then placed in an anaerobic jar, given a gas mixture, and incubated for 24 hrs. E. faecalis also cultured on a BHI medium agar. Then, the agar plate was placed in an anaerobic jar, given the gas mixture, and incubated for 24 hrs. Having grown on the agar surface, the bacteria were removed using the edge of the glass slide. The surface of the medium was then sterilized using chloroform for 30 minutes and left to air dry for 15 minutes to remove the remaining chloroform. Then, E. faecalis were dissolved and incubated for $24 \mathrm{hrs}$. After that, the formed inhibition zone was measured [12]

\section{Plan for data processing and data analysis}

Data analysis was done by comparing the value of the average inhibition zone of $S$. salivarius, and the protein it produces, to the growth of $E$. faecalis isolates in each dish. The value of the average inhibition zone to the growth of E. faecalis was tested through the one-way ANOVA method and was followed by post hoc test. Paired t-test was used to analyzed the potential inhibitory of $S$. salivarius and proteins against $E$. faecalis at varying concentrations. Next, to see the correlation between the increase in the concentration of $S$. salivarius and protein with inhibitory zone widening $E$. faecalis, Pearson's test was used.

\section{RESULTS}

Identification $S$. salivarius and the protein it produces

The results of the identification were done by comparing the morphology of clinical S. salivarius with S. salivarius ATCC 13419, both grown on MSA (Fig. 2). Of the 20 samples (10 saliva samples and 10 samples of dorsal tongue swabs), there were 12 samples of saliva isolates and dorsal tongue smear isolates that had a morphology such as $S$. salivarius colonies on the medium agar. These colonies looked like drops of gum

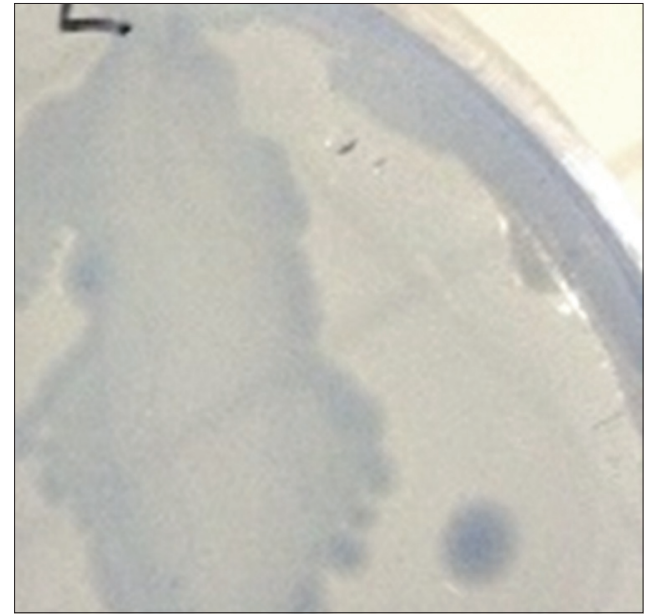

Fig. 1: Morphology culture medium Streptococcus salivarius on mitis Salivarius agar

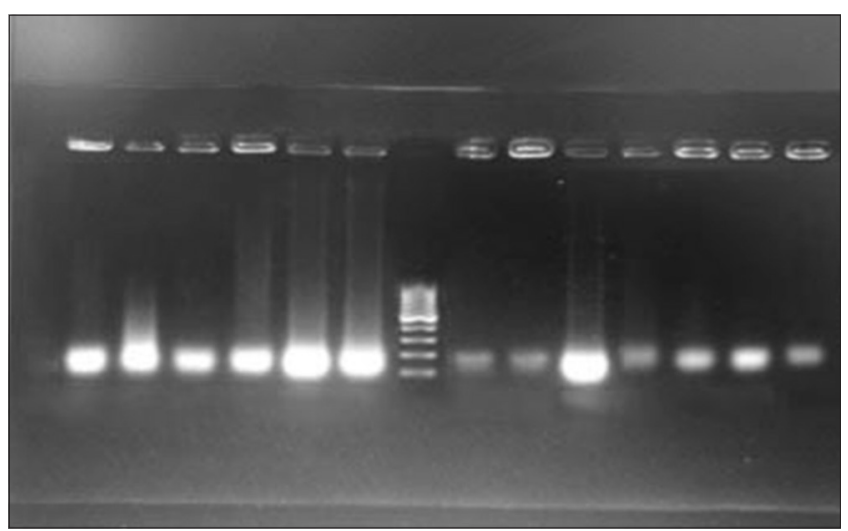

Fig. 2: Polymerase chain reaction results of colonies that have morphology such as Streptococcus salivarius American Type Culture Collection 13419 colonies

(gum-drop like) and were a translucent blue color. Sample saliva isolates and dorsal tongue swab isolates of 10 participants were confirmed using PCR, where $S$. salivarius found in only 6 participants, with 6 samples of saliva and 6 samples of dorsal tongue smears ( $p>0.05$ )

Table 1 shows identification of $S$. salivarius in clinical isolate sources, whereas Fig. 3 shows profile protein $S$. salivarius based on results of SDS-PAGE analysis.

\section{Results of clinical $S$. salivarius' potential to inhibit growth of E. faecalis}

To examine the potential of clinical $S$. salivarius to inhibit the growth of $E$. faecalis, a deferred antagonism method was used. The SDS-PAGE results for the $S$. salivarius samples were similar. Of the 10 S. salivarius colonies, only 2 inhibitory potencies surfaced, as displayed in Fig. 4 . There were significant differences in the total plate count (TPC) $10^{10} \mathrm{CFU} / \mathrm{ml}$ among saliva isolates and dorsal tongue swab isolates $(\mathrm{p}<0.05)$. The inhibition zones produced by $S$. salivarius ATCC 13419 had significant differences with the isolates from the dorsal tongue swabs or saliva samples $(p<0.05)$. The results were reviewed against the inhibition zone on the TPC saliva isolates had a significant difference between the TPC $10^{7}$ $\mathrm{CFU} / \mathrm{ml}$ with TPC $10^{8} \mathrm{CFU} / \mathrm{ml}(\mathrm{p}<0.05)$, whereas among the TPC $10^{8}$ $\mathrm{CFU} / \mathrm{ml}, 10^{9} \mathrm{CFU} / \mathrm{ml}$ and $10^{10} \mathrm{CFU} / \mathrm{mL}$ had no significant difference ( $p>0.05)$. However, unlike the results of inhibition zones for the saliva isolates, the inhibition zones for the dorsal tongue swab isolates had significant differences for all concentrations $(\mathrm{p}<0.05)$. From the analysis of the correlation of the results with TPC inhibition zone for saliva isolates also reviewed and have a moderate level of correlation 
$(\mathrm{p}<0.470)$ and proportional, but the dorsal tongue swab isolates had a high correlation $(\mathrm{p}<0.951)$ and proportional.

\section{Result of protein potential of clinical $S$. salivarius to inhibit growth} of $E$. faecalis

Examination by the agar well diffusion method for the whole-cell protein showed significant differences between the salvia isolates, dorsal tongue smear isolates, and S. salivarius ATCC 13419 samples in $10^{10} \mathrm{CFU} / \mathrm{ml}$. The greatest inhibition zone was produced by the dorsal tongue swab isolates, followed by saliva isolates, and by the S. salivarius ATCC 13419 isolates.

Potential inhibitory protein analysis on whole-cell and protein spent medium

Table 2 shows the average difference between the zone of inhibition of growth when E. faecalis were exposed to whole-cell protein isolates,

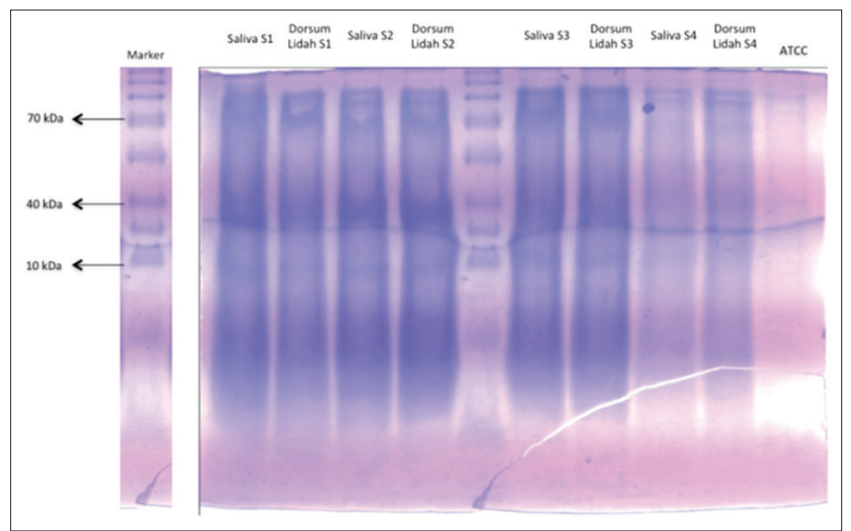

Fig. 3: Profile protein Streptococcus salivarius based on results of sodium dodecyl sulfate polyacrylamide gel electrophoresis analysis

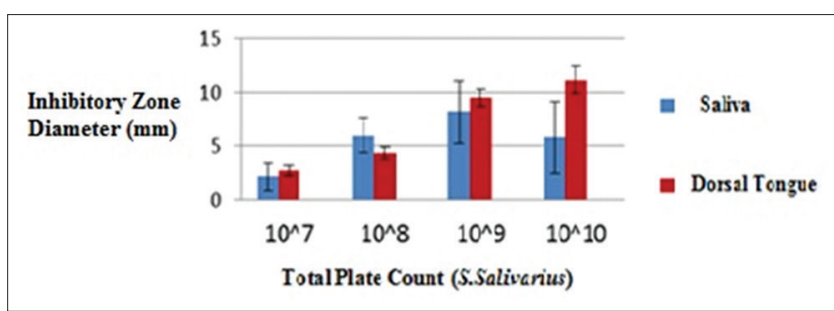

Fig. 4: Potential inhibition of Streptococcus salivarius saliva and dorsal tongue isolates on the growth of Enterococcal faecalis

Table 1: Identification of Streptococcus salivarius in clinical isolate sources

\begin{tabular}{lll}
\hline Isolate sources & \multicolumn{2}{l}{ Streptococcus salivarius } \\
\cline { 2 - 3 } & Positive (\%) & Negative (\%) \\
\hline Saliva & $6(60)$ & $4(40)$ \\
Tongue & $6(60)$ & $4(40)$ \\
\hline
\end{tabular}

and dorsal tongue smear and saliva isolates at a concentration of $2 \times 10^{2} \mathrm{mg} / \mathrm{ml}$. The zone of inhibition in saliva swab isolates was $3.375 \mathrm{~mm}$, and in the dorsal tongue, swab isolates were $8.125 \mathrm{~mm}$. Then, of the overall spent medium proteins that provided the potential for inhibition is spent medium protein in dorsal tongue swab isolates with the greatest concentration of protein $\left(1.05 \times 10^{2} \mu \mathrm{g} / \mathrm{ml}\right)$ with an average of $6 \mathrm{~mm}$ zone of inhibition.

\section{DISCUSSION}

Based on the identification of S. salivarius using MSA and confirmation using PCR, of 10 samples of saliva isolates and 10 samples dorsal tongue swab isolates, S. salivarius was only found in $60 \%$ of the whole sample set, whether saliva isolates or dorsal tongue isolates $(\mathrm{p}>0.05)$. In contrast to research conducted by Amoroso et al., which found that in children aged 12-14 years had the prevalence of $S$. salivarius of 89.31\%. However, it differs from Horz et al., which found newborn oral cavity samples to have only $10 \%$ presence of $S$. salivarius, which then increased to $25-30 \%$ after the babies were 1-month-old. S. salivarius had a tendency to diminish in number while migrating to $2 \%$ of the buccal mucosa, $17 \%$ of the tongue, pharynx, and $30 \%$ of healthy adults $[13,14]$. These differences can be caused by factors such as diet, as observed by Ogawa et al. since dietary factors may change the population of microorganisms in oral cavities [15]. This is also supported in research by Carlsson, who found that the prevalence of $S$. salivarius in the oral cavity of an individual depended on how much sucrose that individual consumed [16].

Further identification of proteins by SDS-PAGE demonstrated colonies of $S$. salivarius saliva isolates and dorsal tongue swab isolates have similar protein compositions. However, S. salivarius ATCC 13419, which served as this study's control, did not produce inhibition zone on E. faecalis. The ability to inhibit bacteria with a classification like $S$. salivarius was supported by Hyink et al. research, which observed that $S$. salivarius $\mathrm{K} 12$ produces salivaricin A2 (SalA2) and B, and act as agents and have proven oral probiotic properties that can be used as antibiotics against Streptococcus pyogenes bacterium targets. Previous study found the isolation of S. salivarius salivaricin 9 NU10 also had the potential to inhibit Gram-positive bacteria, including ATCC 10240 Micrococcus luteus, M. luteus GAB13, Streptococcus equisimilis ATCC 12388, and Corynebacterium spp. GH17. The ability of salivary isolates and dorsal tongue swab isolates of $S$. salivarius to inhibit the growth of E. faecalis is due to the mechanism of quorum sensing. This is supported in research by Konstantinos et al., who concluded that two or more coexisting bacteria will communicate with each other to express a gene in a population using a signal molecule in the form of autoinducers. Konstantinos et al. stated that probiotic agents can inhibit other bacteria in one population using an accessory gene regulator (AGR) quorum sensing system. Quorum sensing AGR-system is a system that is found on the mechanisms of Staphylococcus that cause the bacteria to enlarge their communities by destroying the cells around them [17]. If the mechanism for quorum sensing has occurred, $S$. salivarius would itself produce lantibiotics through autoregulation processes using a two-component regulatory system [4,5,18-20].

The difference in the inhibitory ability between saliva isolates and dorsal tongue swab isolates and S. salivarius ATCC 13419 on the growth of $E$. faecalis was supported in the previous research. They suggested the differences in the virulence of the strains of bacteria are

Table 2: Average zone of inhibition for each concentration isolate protein at each whole-cell uses for agar well diffusion method

\begin{tabular}{|c|c|c|c|c|}
\hline \multicolumn{5}{|l|}{ Protein culture results whole-cell } \\
\hline Protein concentration S. salivarius ATCC $13419(\mu \mathrm{g} / \mathrm{ml})$ & $1.46 \times 10^{2}$ & $1.46 \times 10^{1}$ & 1.46 & $1.46 \times 10^{-1}$ \\
\hline Mean of inhibitory zone & $0 \mathrm{~mm}$ & $0 \mathrm{~mm}$ & $0 \mathrm{~mm}$ & $0 \mathrm{~mm}$ \\
\hline Clinical protein concentration $S$. salivarius (saliva) ( $\mu \mathrm{g} / \mathrm{ml})$ & $2.22 \times 10^{2}$ & $2.22 \times 10^{1}$ & 2.22 & $2.22 \times 10^{-1}$ \\
\hline Mean of inhibitory zone & $3.375 \mathrm{~mm}$ & $0 \mathrm{~mm}$ & $0 \mathrm{~mm}$ & $0 \mathrm{~mm}$ \\
\hline Clinical protein concentration $S$. salivarius (dorsal tongue) $(\mu \mathrm{g} / \mathrm{ml})$ & $2.28 \times 10^{2}$ & $2.28 \times 10^{1}$ & 2.284 & $2.28 \times 10^{-1}$ \\
\hline Mean of inhibitory zone & $8.125 \mathrm{~mm}$ & $0 \mathrm{~mm}$ & $0 \mathrm{~mm}$ & $0 \mathrm{~mm}$ \\
\hline
\end{tabular}

S. salivarius: Streptococcus salivarius, ATCC: American Type Culture Collection 
due to differences in the protein produced by clinical and laboratory strains. Kim et al. used samples of the laboratory and clinical $E$. faecalis strains, which were tested using multilocus sequence typing by seven housekeeping genes. They found that the laboratory E. faecalis strain was virulently weak compared to other strains $[21,22]$. In the deferred antagonism method, results showed colonies of $S$. salivarius saliva isolates and dorsal tongue swab isolates, as seen in Fig. 4. Isolates from dorsal tongue swabs had a high correlation in each resulting $E$. faecalis inhibition zone against bacterial count $10^{7} \mathrm{CFU} / \mathrm{ml}, 10^{8} \mathrm{CFU} / \mathrm{ml}, 10^{9}$ $\mathrm{CFU} / \mathrm{ml}$, and $10^{10} \mathrm{CFU} / \mathrm{ml}$ based on statistical analysis ( $\mathrm{p}=0.951$ ). In contrast to the saliva isolates, the result of the inhibition zone E. faecalis by the number of bacteria have statistical results with a moderate level of correlation $(p=0.470)$. These results were also supported by another research on the concentration effects of antibiotics. In this study, inhibition zones were affected by the number of bacteria applied to E. faecalis, especially on S. salivarius derived from dorsal tongue smear isolates [23]. Then, referring to S. salivarius ATCC 13419, the study's positive control, the second colony of clinical trials showed the potential to inhibit the growth of $E$. faecalis. There was also a difference between the zone drag generated by the control and each $S$. salivarius colony derived from the dorsal tongue swabs and saliva at a concentration of $10^{10} \mathrm{CFU} / \mathrm{ml}(\mathrm{p}<0.05)$. The effective capability of inhibiting the growth of $E$. faecalis isolates minimum visible from the dorsal tongue and saliva swabs with the number of $10^{7} \mathrm{CFU} / \mathrm{ml}$. Visible also the ability of inhibitory S. salivarius K12 effective on research conducted by Ishijima on the prevention of $C$. albicans adhesion to plastic cups when the number of S. salivarius $\mathrm{K} 12$ as much as $3.75 \times 10^{8} \mathrm{CFU} / \mathrm{ml}$ [24].

The two colonies secreted proteins and whole-cell protein isolates were tested through the agar well diffusion method. Using this method, the interaction between a proteins produced by $S$. salivarius against the formation of inhibition zone on the growth of E. faecalis was analyzed. Since the whole-cell protein that was extracted from $S$. salivarius clinical cultured in BHI broth was allowed to seep in order that already contains $E$. faecalis. From the results obtained only inhibitory zone at a concentration of $2 \times 10^{2} \mathrm{mg} / \mathrm{ml}$ in saliva isolates and isolates smear dorsal tongue. Similarly, the protein was isolated from the supernatant of $S$. salivarius isolates and S. salivarius ATCC 13419 isolates produced only inhibition zone from the dorsal tongue smear with a concentration of $1.05 \times 10^{2} \mu \mathrm{g} / \mathrm{ml}$. Lantibiotics are the secondary protein produced in the stationary phase, and $S$. salivarius is a lactic acid bacterium that produces the protein. Lantibiotics produced by lactic acid bacteria have two types, Type A is lantibiotic with linear shape and Type B has a globular shape. The difference in the types of lantibiotic is also evident from the work process described in the study conducted by Hasper [6]. Lantibiotic Type A destroys the target cell's membrane integrity and lantibiotic type B interferes with enzymes that work in the biosynthesis of the cell wall [25]. The diversity of the types of lantibiotic proteins depend on the type of S. salivarius. The differences in the results of inhibition zone produced by saliva and dorsal tongue swab isolates may be due to differences in the types of protein produced by the different isolates [26].

The difference in the working mechanisms of the research is also supported by Hasper et al. in their research on lantibiotic proteins. They found that the mechanism of action of the protein with a small peptide does not require protein receptors on the membrane of target cells but directly work on the molecule biosynthesis, namely, lipid II [6]. Another mechanism that is different is expressed also by previous study indicating that the mechanism of Type B lantibiotic as seen in SalB. SalB works by inducing the accumulation of a precursor (UDP-MurNAcpentapeptide) from the end of the cell wall, thus causing obstacles to biosynthesis within the cell walls [26]. The observed differences are also due to its antimicrobial activity. Lantibiotic and lantipeptida consist of four subclasses, where the subclass III and IV form from a peptide that resembles lantibiotic, such as peptide morphogenetic, and lantipeptida have the low antimicrobial ability $[27,28]$.

Another study also stated that the correlation between the concentration lantibiotic is significant in disrupting the integrity of the cell membrane
M. luteus ATCC 10240 with an increase in MFI by 4 or 5 times more [5]. In the study conducted by Upton et al., some lantibiotic proteins have autoregulation properties such as SalA and SalA1. It requires induction of SalA into the bacterium with mutated SalB [29]. The possibility of no formation of several agents lantibiotic, such as SalA, was likely to occur in this study because inducer agents such as salivaric in 9 or SalA, were not used. Dagim suggests the failure to inhibit most lantibiotic proteins was more effective when exposed to Gram-positive bacteria. The resulting inhibition zone of deferred antagonism method was more successful than the agar well diffusion method. In addition, the poor results of the agar well diffusion method may also be due to lack of the protein concentration of the spent medium and whole-cells applied into each well. Deficiencies in the control due to time constraints of the experiments also become one of the problems in determining the potential inhibition potency.

\section{CONCLUSION}

There is potential for S. salivarius isolates from the dorsal tongue and saliva swabs to inhibit the growth of $E$. faecalis, with greater potential from the dorsal tongue smear than the saliva isolates. Not all proteins are derived from S. salivarius isolates inhibited the growth of bacteria $E$. faecalis.

\section{REFERENCES}

1. Mack DR. Probiotics-mixed messages. Can Fam Physician 2005;51:1455-7, 1462-4.

2. Van Niel CW, Feudtner C, Garrison MM, Christakis DA. Lactobacillus therapy for acute infectious diarrhea in children: A meta-analysis. Pediatrics 2002;109(4):678-84.

3. Nouaille S, Ribeiro LA, Miyoshi A, Pontes D, Le Loir Y, Oliveira $\mathrm{SC}$, et al. Heterologous protein production and delivery systems for Lactococcus lactis. Genet Mol Res 2003;2(1):102-11.

4. Di Pierro F, Donato G, Fomia F, Adami T, Careddu D, Cassandro C, et al. Preliminary pediatric clinical evaluation of the oral probiotic Streptococcus salivarius $\mathrm{K} 12$ in preventing recurrent pharyngitis and/or tonsillitis caused by Streptococcus pyogenes and recurrent acute otitis media. Int J Gen Med 2012;5:991-7.

5. Barbour A, Philip K, Muniandy S. Enhanced production, purification, characterization and mechanism of action of salivaricin 9 lantibiotic produced by Streptococcus salivarius NU10. PLoS One 2013;8(10):e77751.

6. Hasper HE, Kramer NE, Smith JL, Hillman JD, Zachariah C, Kuipers OP, et al. An alternative bactericidal mechanism of action for lantibiotic peptides that target lipid II. Science 2006;15:1636-7.

7. RISKESDAS. Riset Kesehatan Dasar. Jakarta: Badan Penelitian dan Pengembangan Kesehatan Kementerian Kesehatan RI; 2013.

8. Vinotha T, Geetha RV. Natural remedy to prevent tooth decay: A review. Asian J Pharm Clin Res 2015;8(1):32-3.

9. Yadav R, Yadav SK. Dental disease and its cure: A review. Asian J Pharm Clin Res 2013;6(2):16-20.

10. Stuart CH, Schwartz SA, Beeson TJ, Owatz CB. Enterococcus faecalis: Its role in root canal treatment failure and current concepts in retreatment. J Endod 2006;32(2):93-8.

11. Bosch-Aranda ML, Canalda-Sahli C, Figueiredo R, Gay-Escoda C. Complications following an accidental sodium hypochlorite extrusion: A report of two cases. J Clin Exp Dent 2012;4(3):e194-8.

12. Wescombe PA, Upton M, Dierksen KP, Ragland NL, Sivabalan S, Wirawan RE, et al. Production of the lantibiotic salivaricin A and its variants by oral streptococci and use of a specific induction assay to detect their presence in human saliva. Appl Environ Microbiol 2006;72(2): 1459-66.

13. Horz HP, Meinelt A, Houben B, Conrads G. Distribution and persistence of probiotic Streptococcus salivarius K12 in the human oral cavity as determined by real-time quantitative polymerase chain reaction. Oral Microbiol Immunol 2007;22(2):126-30.

14. Amoroso P, Ávila FA, Gagliardi CM. Prevalence of different streptococci species in the oral cavity of children and adolescents. Braz J Oral Sci 2003;2(4):164-8

15. Ogawa A, Furukawa S, Fujita S, Mitobe J, Kawarai T, Narisawa N, et al. Inhibition of Streptococcus mutans biofilm formation by Streptococcus salivarius FruA. Appl Environ Microbiol 2011;77(5):1572-80.

16. Carlsson J. Effect of diet on presence of Streptococcus salivarius in dental plaque and saliva. Odontol Revy 1965;16(4):336-47.

17. Yarwood JM, Schlievert PM. Quorum sensing in Staphylococcus 
infections. J Clin Invest 2003;112(11):1620-5.

18. Papadimitriou K, Zoumpopoulou G, Foligné B, Alexandraki V, Kazou M, Pot B, et al. Discovering probiotic microorganisms: In vitro, in vivo, genetic and omics approaches. Front Microbiol 2015;6:58

19. Waters CM, Bassler BL. Quorum sensing: Cell-to-cell communication in bacteria. Annu Rev Cell Dev Biol 2005;21:319-46.

20. Gross R, Beier D, Marina A, Keilberg D, Huntley S, Søgaard-andersen L. Two-Component Systems in Bacteria. Germany: Caister Academic Press, University of Würzburg; 2012.

21. Kim EB, Kopit LM, Harris LJ, Marco ML. Draft genome sequence of the quality control strain Enterococcus faecalis ATCC 29212. J Bacteriol 2012;194(21):6006-7.

22. Sturme MH, Nakayama J, Molenaar D, Murakami Y, Kunugi R, Fujii $\mathrm{T}$, et al. An agr-like two-component regulatory system in Lactobacillus plantarum is involved in production of a novel cyclic peptide and regulation of adherence. J Bacteriol 2005;187(15):5224-35.

23. Levison ME, Levison JH. Pharmacokinetics and pharmacodynamics of antibacterial agents. Infect Dis Clin North Am 2009;23(4):791-815, vii.
24. Ishijima SA, Hayama K, Burton JP, Reid G, Okada M, Matsushita Y, et al. Effect of Streptococcus salivarius K12 on the in vitro growth of Candida albicans and its protective effect in an oral candidiasis model. Appl Environ Microbiol 2012;78(7):2190-9.

25. Chatterjee C, Paul M, Xie L, van der Donk WA. Biosynthesis and mode of action of lantibiotics. Chem Rev 2005;105(2):633-84.

26. Barbour A, Tagg J, Abou-Zied OK, Philip K. New insights into the mode of action of the lantibiotic salivaricin B. Sci Rep 2016;6:31749.

27. Drider D, Rebuffat S. Prokaryotic antimicrobial peptides. Prokaryotic Antimicrobial Peptides: From Genes to Applications. New York: Springer; 2011.

28. Birri DJ, Brede DA, Nes IF. Salivaricin D. A novel intrinsically trypsinresistant lantibiotic from Streptococcus salivarius 5M6c isolated from a healthy infant. Appl Environ Microbiol 2012;78(2):402-10.

29. Upton M, Tagg JR, Wescombe P, Jenkinson HF. Intra- and interspecies signaling between Streptococcus salivarius and Streptococcus pyogenes mediated by SalA and SalA1 lantibiotic peptides. J Bacteriol 2001;183(13):3931-8. 